\title{
Bacteriome in Ticks Collected from Domestic Livestock in Kenya
}

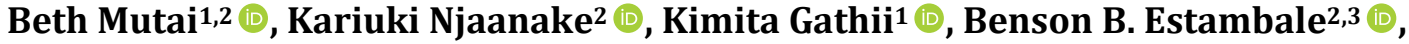 \\ John N. Waitumbi ${ }^{*}$ (i)
}

${ }^{1}$ US Army Medical Research Directorate-Africa/Kenya Medical Research Institute, Kisumu, Kenya

${ }^{2}$ Department of Medical Microbiology, University of Nairobi, Nairobi, Kenya

${ }_{3}^{3}$ Jaramogi Oginga Odinga University of Science and Technology, Bondo, Kenya

Email: ^john.waitumbi@usamru-k.org

How to cite this paper: Mutai, B., Njaanake, K., Gathii, K., Estambale, B.B. and Waitumbi, J.N. (2022) Bacteriome in Ticks Collected from Domestic Livestock in Kenya. Advances in Microbiology, 12, 67-82. https://doi.org/10.4236/aim.2022.122006

Received: December 2, 2021

Accepted: February 15, 2022

Published: February 18, 2022

Copyright $\odot 2022$ by author(s) and Scientific Research Publishing Inc. This work is licensed under the Creative Commons Attribution International License (CC BY 4.0).

http://creativecommons.org/licenses/by/4.0/

\begin{abstract}
Background: Metagenomics approaches are increasingly being utilized as "dipstick" for microbial carriage. In this study, $16 \mathrm{~S}$ rRNA metagenomics was used to probe for microbial community that resides in the ticks, those they pick from the environment, wildlife and livestock and to identify potential tick borne zoonoses. Methods: Tick DNA from 463 tick pools collected from domestic animals between 2007 and 2008 were amplified with primers that target the 16S rRNA V3-V4 domain and then sequenced on Illumina Miseq platform using 300 cycles version 3 kits. Ticks were pooled according to species and animal from which they were collected. A non-target control was used to track laboratory contaminants. Sequence data were analyzed using Mothur v1.3 pipeline and R v3.3.1 software and taxonomy determined using SILVA rRNA database. Shannon diversity index was used to compute bacterial diversity in each tick species before computing the means. Results: A total of 645 bacteria genera grouped into 27 phyla were identified. Four phyla contributed $97.4 \%$ of the $36,973,934$ total sequences. Proteobacteria contributed $61.2 \%$ of these sequences that tarried to $33.8 \%$ genera, compared to 15.9\% (23.4\% genera) for Firmicutes, 15.6\% (20\% genera) for Actinobacteria and $4.7 \%$ ( $11.6 \%$ genera) for Bacteroidetes. The remaining 23 phyla only contributed $2.6 \%$ of the sequence reads (11.2\% genera). Amongst the 645 genera, three groups were discernible, with the biggest group comprised commensals/symbionts that contributed $93.6 \%$ of the genera, but their individual sequence contribution was very low. Group two comprised genera that are known to contain pathogenic species, with Coxiella contributing 15,445,204 (41.8\%) sequences, Corynebacterium (13.6\%), Acinetobacter (4.3\%), Staphylococcus (3.9\%), Bacillus (2.7\%) and Porphyromonas (1.6\%), Ralstonia (1.5\%), Streptococcus (1.3\%), Moraxella (1.3\%), amongst others. Group three com-
\end{abstract}


prised genera known to contain tick borne zoonotic pathogens (TBZ): Rickettsiae, Anaplasma, Francisella, Ehrlichia, Bartonella and Borrelia. Individually the TBZ contributed $<1 \%$ of the sequences. By Shannon diversity index, Amblyomma variegatum carried the least diverse bacteria (mean Shannon diversity index of $2.69 \pm 0.92$ ) compared to $3.79 \pm 1.10$ for $A$. gemma, $3.71 \pm 1.32$ for $A$. hebraeum, $4.15 \pm 1.08$ for other Amblyomma spp, $3.79 \pm$ 1.37 for Hyalomma truncatum, $3.67 \pm 1.38$ for other Hyalomma spp, $3.86 \pm$ 1.27 for Rhipicephalus annulatus, $3.56 \pm 1.21$ for $R h$. appendiculatus, and $3.65 \pm 1.30$ for $R h$. Pulchellus, but the difference was not significant ( $\mathrm{p}=$ 0.443). Conclusion: This study illustrates the utility of $16 \mathrm{~S}$ rRNA metagenomics in revealing the complexity of bacteria communities that reside and/or transit through the tick having been picked from the environment, livestock and/or wild animals, some with potential to cause zoonoses.

\section{Keywords}

Livestock, Ticks, Bacteriome, Tick-Borne Zoonoses, 16S rRNA, Next Generation Sequencing

\section{Introduction}

Worldwide, ticks are considered second to mosquitoes in their ability to transmit disease causing pathogens, and are the most relevant disease vectors for domestic and wild animals [1]. Among the tick borne zoonotic pathogens (TBZ) vectored by ticks are tick-borne encephalitis virus (TBEV), Crimean Congo hemorrhagic fever virus (CCHFV), Babesia spp., Rickettsia, Anaplasma, Francisela, Borrelia, Bartonella, Coxiella and Ehrlichia [2], which they can transmit transstadially and in some cases transovarially [3]. In addition, an individual tick can harbor multiple pathogens which could be co-transmitted during feeding [4]. Other microbes co-exist in ticks as endosymbionts or commensals, and have been reported to influence vector survival and pathogen transmission fitness [5]. Until the advent of next generation sequencing (NGS), methods for detection targeted known pathogens [6], making routine pathogen discovery efforts harder. NGS is pathogen agnostic and allows unbiased detection of sequences in a sample that can then be analyzed bioinformatically for matches in microbial gene repositories.

For this study, 16S rRNA gene, which is universally present in all bacteria, was used for identification of bacteria communities in ticks. The gene comprises 9 hyper-variable regions (V1 to V9) that are interspaced by conserved regions [7] [8]. Although the variable regions have considerable sequence diversity, no single region can differentiate all bacteria and a combination of 2 or more regions is recommended [7] [9] [10]. A combination of V3-V4 region that covers 460 base-pairs (bp) increases the accuracy of taxonomic classification to genus level compared to other variable regions [10] [11] [12]. Using these regions, the Illumina 300 cycles sequencing kit can be used to process $460 \mathrm{bp}$ paired-ends reads 
[12].

The 16S rRNA approach has been used for detection of pathogenic bacteria and symbionts in ticks [13] [14] [15] [16] [17]. These studies have reported up to $80 \%$ dominance of Proteobacteria followed by Actinobacteria, Bacteroidetes or Firmicutes [13] [14]. Potentially pathogenic genera that have been identified in these studies include Anaplasma, Coxiella, Ehrlichia, Rickettsia, Borrelia, Acinetobacter, Burkholderia and Staphylococcus among others [14] [15] [16]. In addition to harboring bacteria of medical relevance, ticks also carry endosymbionts such as Midichloria mitochloria, Wolbachia spp., Neoehrlichia spp., Rickettsiella. Endosymbionts very similar to tick-transmitted pathogens, including Coxiella-like, Rickettsia-like, or Francisella-like [13] [17] [18] that are said to provide additional nutrients lacking in blood meals have also been identified [19] [20]. Thus, 16S rRNA metagenomics is a useful approach for analyzing the complexity of bacteria community in ticks, as well as identifying potential TBZ.

This study used 16S rRNA metagenomics to examine bacterial community in ticks that were collected from livestock. We highlight the complexity of tick microbiome that they can acquire from the environment, livestock, wildlife or humans, some of them with potential to cause zoonoses.

\section{Method}

\subsection{Ethics Statement}

This study used archived genomic DNA from ticks that were collected from domestic livestock as detailed before [21]. The tick samples were collected under an animal use protocol SSC\#1248 that was reviewed and approved by the Animal Use Committee of the Kenya Medical Research Institute.

\subsection{Sample Acquisition and Study Sites}

Tick samples had been collected from 333 cattle, 112 Sheep and 18 goats as previously described [21]. Briefly, ticks were collected from domestic animals presented for slaughter to major slaughterhouses in Nairobi (Athi River Kenya Meat Commission [KMC]) and Mombasa (Kibarani, Uwanja wa Ndege, Mariakani and Kasemeni) between November, 2007 and September, 2008. Up to 10 ticks were collected from each animal that was infested with ticks and placed in $1.5-\mathrm{mL}$ vials containing $70 \%$ ethanol, then transported to the laboratory. Ticks were identified taxonomically using the standard taxonomic keys [22]. Ticks of the same species and from the same domestic animal were pooled together and placed in a $1.5-\mathrm{mL}$ vial containing $70 \%$ ethanol and stored at $-80^{\circ} \mathrm{C}$.

\subsection{Genomic DNA Isolation from Ticks}

Tick pools were re-hydrated, homogenized using a pestle and mortar and then suspended in $1 \mathrm{~mL}$ of phosphate buffered saline as described before [20]. DNA was extracted from $200 \mu \mathrm{l}$ of the homogenate using a Qiagen QIAamp DNA Mini Kit according to manufacturer's instructions (Qiagen Inc., Valencia, CA). 
Genomic DNA was eluted in $200 \mu \mathrm{L}$ and stored at $-80^{\circ} \mathrm{C}$ until testing was performed.

\subsection{Amplification of $16 S$ rRNA V3-V4 Region, Library Preparation and Sequencing}

Bacteria DNA in the ticks was amplified with primers targeting $16 \mathrm{~S}$ rRNA V3-V4 region as described earlier [23]. The primers were tagged with Illumina sequencing adapters. PCR water was used as non-target control and was included in each run to track laboratory contaminants. Briefly, PCR was performed in a total volume of $25 \mu \mathrm{L}$ that contained $2.5 \mu \mathrm{L}$ of genomic DNA, $5 \mu \mathrm{L}$ of forward and reverse primers, each at a final concentration of $1 \mu \mathrm{M}$ and 12.5 $\mu \mathrm{L}$ of NEBNext High-Fidelity 2X PCR Master Mix (New Englands BioLabs, MA USA) at $95^{\circ} \mathrm{C}$ initial denaturation for $3 \mathrm{~min}$, followed by 25 cycles of $95^{\circ} \mathrm{C}$ for 30 $\mathrm{s}, 62.3^{\circ} \mathrm{C}$ for $30 \mathrm{~s}$, and $72^{\circ} \mathrm{C}$ for $30 \mathrm{~s}$, and a final extension at $72^{\circ} \mathrm{C}$ for $5 \mathrm{~min}$. Amplicons were purified with Agencourt AMPure XP beads (Beckman Coulter Genomics, CA USA) according to the manufacturer's protocol.

A dual indexing PCR to allow multiplexing of samples was done using $5 \mu \mathrm{L}$ of purified amplicons, $5 \mu \mathrm{L}$ of Nextera XT i7 Index Primer, $5 \mu \mathrm{L}$ of Nextera XT i5 Index Primer (Illumina, CA USA), $25 \mu \mathrm{L}$ of NEBNext High-Fidelity 2X PCR Master Mix (New Englands BioLabs, MA USA) and $10 \mu \mathrm{L}$ of PCR grade water (Thermo Fisher Scientific, MA USA), with thermocycling at $95^{\circ} \mathrm{C}$ for $3 \mathrm{~min}$, followed by 12 cycles of $95^{\circ} \mathrm{C}$ for $30 \mathrm{~s}, 55^{\circ} \mathrm{C}$ for $30 \mathrm{~s}$, and $72^{\circ} \mathrm{C}$ for $30 \mathrm{~s}$, and a final extension at $72^{\circ} \mathrm{C}$ for $5 \mathrm{~min}$. Constructed libraries were purified using Agencourt AMPure XP beads (Beckman Coulter Genomics, MA USA) according to manufacturer's protocol. The libraries were quantified on Qubit Flourometer 2.0 using Qubit dsDNA HS Assay kit (ThermoFsher Scientific, MA USA). Libraries were normalized and pooled to $4 \mathrm{nM}$ based on Qubit values. Pooled samples were denatured and diluted to a final concentration of $10 \mathrm{pM}$ and spiked with 10\% PhiX (Illumina, CA USA). 96 samples that included appropriate controls were multiplexed in each sequencing run and paired end sequenced using MiSeq Reagent Kit V3 on the Illumina MiSeq System (Illumina, CA USA).

\section{Data Analysis}

The 16S rRNA MiSeq sequences output were de-multiplexed and adapters trimmed using the MiSeq reporter software version 2.6.3 (Illumina, CA USA). Mothur pipeline (version 1.35) was used for paired end reads contig assembly, sequence quality filtering, chimera removal and taxonomic assignment [24]. In brief, contigs containing ambiguous bases, and those with lengths $<350 \mathrm{bp}$ (bp) or greater than $466 \mathrm{bp}$ were discarded. Sequences were then aligned to a customized V3-V4 region on the SILVA database [25], followed by merging sequences that were not more than 2 bp different from each other using the pre-cluster command in Mothur [24]. The merged sequences were then filtered for chimeras before taxonomic assignment against the customized V3-V4 SILVA database [24] using Bayesian classifier with 80\% confidence [24] [25]. Unas- 
signed operational taxonomic unit (OTU) and those assigned to Chloroplast, Mitochondria, Archaea, and Eukaryote were discarded. Samples with less than $1000(\mathrm{n}=40)$ sequences were excluded from downstream analysis as small library sizes often conceal biologically meaningful results [26]. Taxa detected in the non-template control were censored from the tick sample dataset [27].

Statistical analysis and data visualization were done from OTU tables generated by Mothur on R software environment version 4, with Phyloseq, vegan and ggplot2 statistical adds-on [28] [29] [30] [31]. To down sample the data for alpha diversity calculation and account for unequal sequencing between samples, rarefaction was done using rarefy_even_depth command in phloseq with replacement [32]. The rarefied data was used to determine Shannon diversity index by first determining bacterial diversity for each tick species before computing the mean [33].

\section{Results}

\subsection{Tick Samples}

In total, 463 tick pools belonging to three tick genera, 25\% $(\mathrm{n}=113)$ Amblyomma (A. gemma, A. hebraeume, A. variegatum and Amblyomma spp.), 13\% ( $\mathrm{n}=$ 62) Hyalomma (H. truncatum, other Hyalomma spp.) and 62\% $(\mathrm{n}=288)$ Rhipicephalus (Rh. Annulatus, Rh. appendiculatus and Rh. Pulchellus) were accessed in this study.

\subsection{S rRNA Sequencing Results}

Of the 463 tick pools, 400 had their paired end sequences assembled and yielded a total of 97,993,917 contigs. The lowest number of sequence contigs in a sample was 237 , and the highest was 4,252,150. After quality filtering, collapsing duplicate sequences, removing chimeras and non-bacterial sequences, 41,500,930 unique sequences remained and were used for taxonomic assignment. Using prevalence method in the "decontan" command within R package, 6 OTUs were identified as contaminants and removed from the dataset. 40 tick pools with library size of $<1000$ sequences were dropped from downstream analysis, leaving a total of 360 tick pools with $36,973,934$ sequence reads.

\subsection{Bacterial Community Detected in Ticks}

645 unique OTUs (genera) were identified in ticks representing over 89\% $(36,973,934 / 41,500,930)$ of the sequences that passed the QC after quality filtering. The bacteria genera grouped into 27 phyla and as shown in Figure 1, Proteobacteria contributed the majority $(61.2 \%)$ of the sequences that tarried to 33.8\% OTUs, $15.9 \%$ for Firmicutes (23.4\% OTUs), 15.6\% for Actinobacteria (20\% OTUs) and $4.7 \%$ for Bacteroidetes (11.6\% OTUs). The remaining 23 phyla only contributed $2.6 \%$ of the sequence reads ( $11.2 \%$ OTUs) that included Fusobacteria (0.7\%), TM7 (Saccharibacteria) (0.5\%), Verrucomicrobia (0.3\%), Acidobacteria (0.2\%), Deinococcus-Thermus (0.2\%), Planctomycetes $(0.2 \%)$, 


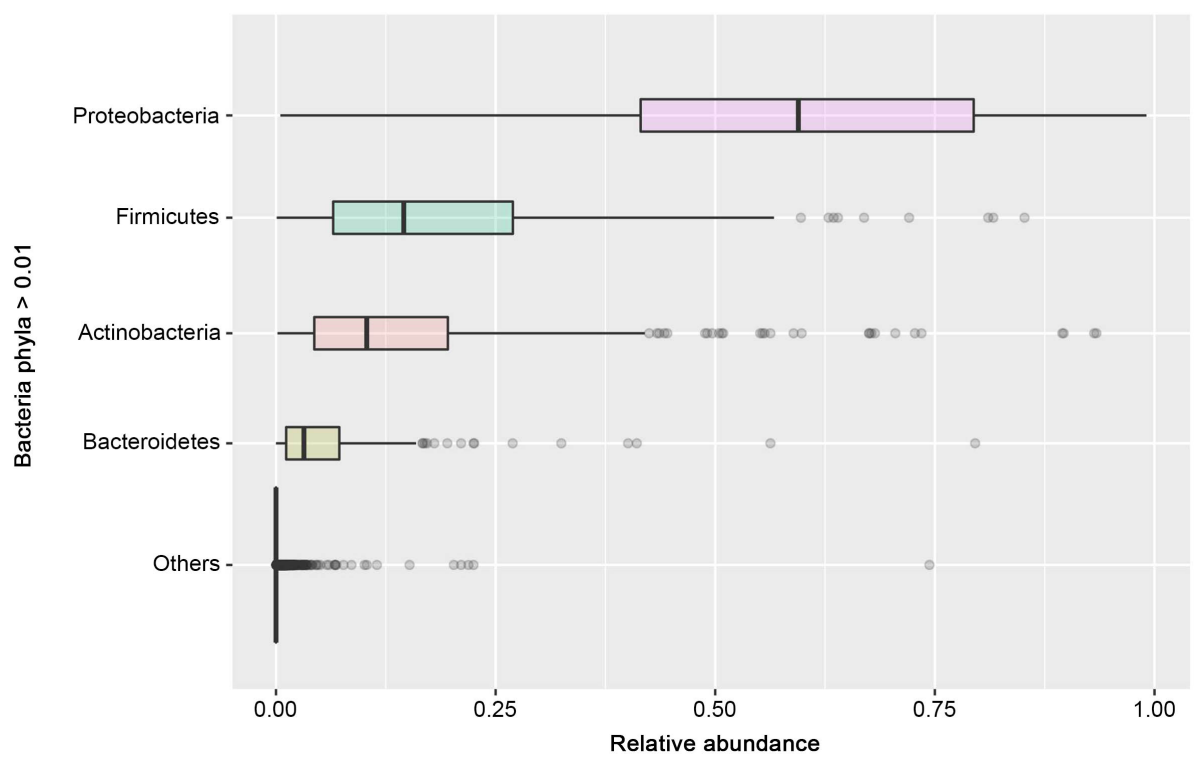

Figure 1. Box plots showing the 27 bacterial phyla detected in ticks with relative abundance $>0.01 \%$. Four phyla contributed $97.4 \%$ of the sequences, of which Proteobacteria dominated (61.2\%) followed by Firmicutes (15.9\%), Actinobacteria (15.6\%) and Bacteroidetes $(4.7 \%)$. The remaining 23 phyla are grouped together as "Others" and contributed $2.6 \%$ of the total sequences.

Chloroflexi (0.1\%). Others with less than $0.1 \%$ sequence reads included OD1 (Parcubacteria), Tenericutes, Gemmatimonadetes, Armatimonadetes, Spirochaetes, Aquificae, SR1 (Absconditabacteria), Lentisphaerae, BRC1, Chlamydiae, Nitrospira, Chlorobi, Synergistetes, Fibrobacteres, WS3 and Elusimicrobia.

Of the 645 genera identified, three groups were discernible (Figure 2): commensals/symbionts (Figure 2, panel A) that contributed $93.6 \%$ of the OTUs, but their individual sequence contributions were very low (25\% out of 36,973,934 sequences). Commensals in this group included Cloacibacterium, Aquabacterium, Schlegelella, Tepidimonas, Aerococcus, Enhydrobacter, Acidovorax, Proteus, Micrococcus, and Fusobacterium among others. Group two comprised genera known to contain pathogenic species that were probably picked by the ticks from the livestock and/or environment (Figure 2, panel B). In this group, Coxiella dominated, contributing 41.8\% (15,445,204 out of $36,973,934)$ of the sequences, Corynebacterium 13.6\%, Acinetobacter $4.3 \%$, Staphylococcus 3.9\%, Bacillus 2.7\%, Porphyromonas 1.6\%, Ralstonia 1.5\%, Streptococcus $1.3 \%$, and Moraxella $1.3 \%$ (Figure 2 Panel B). Others that contributed $<0.1 \%$ of total sequences included Burkholderia, Klebsiella, Escherichia, Shigella, Achromobacter, Haemophilus, Legionella, Campylobacter, Treponema, Elizabethkingia, Mycoplasma, Bordetella, Vibrio and Brucella (Figure 2 Panel B). Group three comprised genera known to contain tick borne zoonotic pathogens (TBZ): Rickettsiae, Anaplasma, Francisella, Erlichia, Bartonella and Borrelia. Individually the TBZ contributed $<1 \%$ of the sequences (Figure 2 Panel C). 


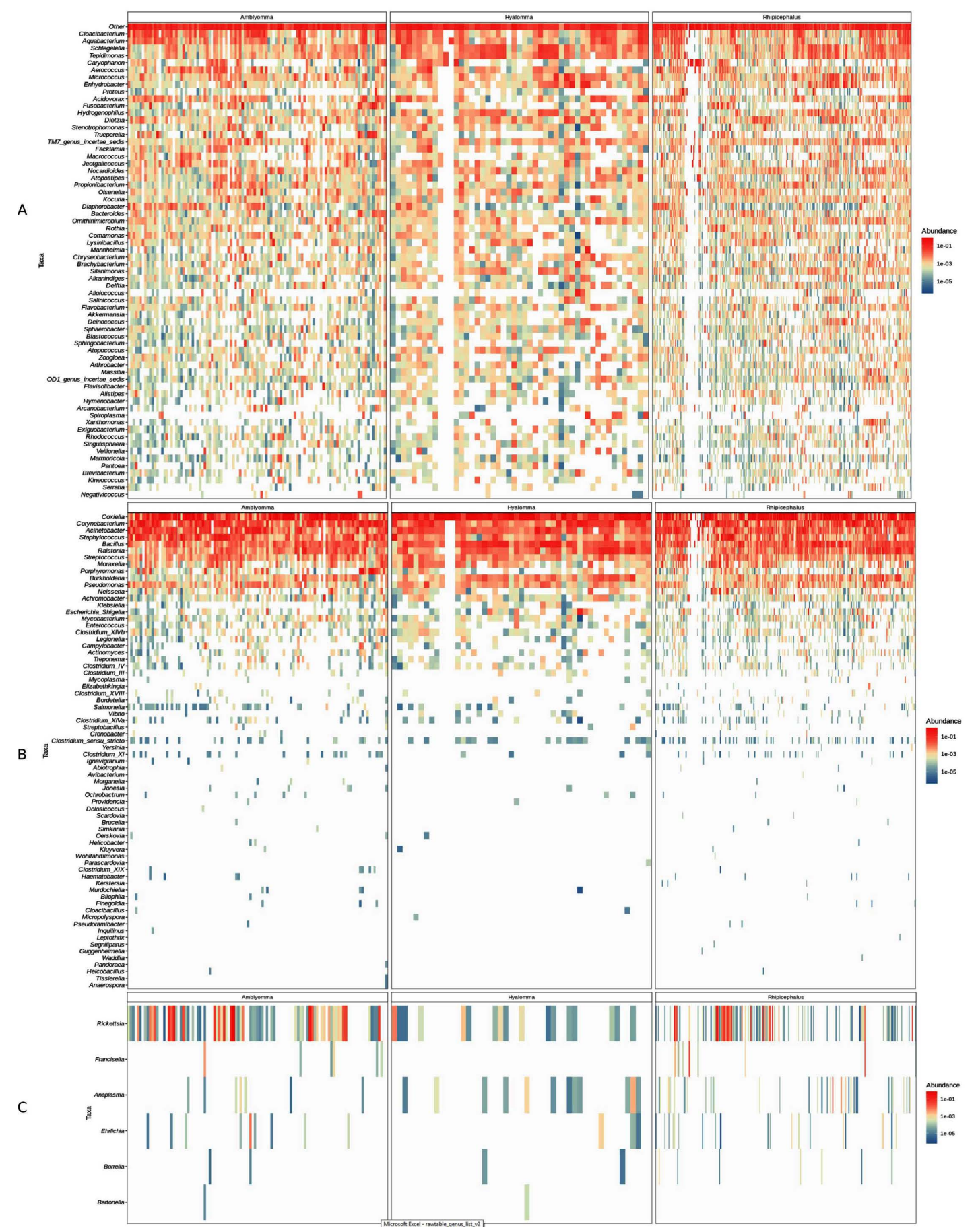

Figure 2. Heat map showing the three groups of bacteria that were identified in different tick genera. Panel A shows taxa that are probably commensals/symbiotic or from the environment appearing at abundance $>1 \%$ ). The commensals/symbionts group dominated, contributing $93.6 \%$ of the OTUs, but their individual sequence contribution was very low. Group two comprised genera that are known to have pathogenic species (Panel B): Coxiella dominated in this group at 41.8\%, followed by Corynebacterium (13.6\%), Acinetobacter (4.3\%), Staphylococcus (3.9\%), Bacillus (2.7\%) and Porphyromonas (1.6\%), Ralstonia (1.5\%), Streptococcus (1.3\%), Moraxella (1.3\%), amongst others. Group three comprised tick borne zoonotic pathogens (Panel C): Rickettsiae, Anaplasma, Francisella, Ehrlichia, Bartonella and Borrelia and individually contributed $<1 \%$ of the sequences. The $\mathrm{X}$-axis represents the samples from the different tick genus. The Y-axis represents microbial taxa at the genus level, ordered by hierarchical clustering. Red color indicates a greater number of reads of that bacterial genus and dark blue color indicates less reads. White boxes indicate samples without sequence reads. 


\subsection{Diversity of Tick Bacterial Community}

A total of 37,344,683 sequences from 360 samples were rarefied to 1220 reads per samples resulting in 381,726 sequences that were used to determine the Shannon diversity index. As shown in Figure 3, the diversity index ranged from 2.69 to 4.15. A. variegatum showed the least diversity (mean Shannon diversity index of $2.69 \pm 0.92$ ) compared to $3.79 \pm 1.10$ for $A$. gemma, $3.71 \pm 1.32$ for $A$. hebraeum, $4.15 \pm 1.08$ for other Amblyomma spp, $3.79 \pm 1.37$ for Hyalomma truncatum, $3.67 \pm 1.38$ for other Hyalomma spp, $3.86 \pm 1.27$ for Rhipicephalus annulatus, $3.56 \pm 1.21$ for Rh. appendiculatus, and $3.65 \pm 1.30$ for Rh. Pulchellus, but the difference was not significant $(\mathrm{p}=0.443)$.

\section{Discussion}

This study presents data on bacterial communities associated with ticks of the genus Rhipicephalus, Amblyomma and Hyalomma that were collected from domestic animals (cattle, sheep and goats). Ticks belonging to these genera feed on multiple hosts, including wildlife and domestic livestock, in addition to being homophilic, vector multiple zoonotic pathogens [3], and are therefore very important component of "One Health". To the best of our knowledge, our study is the first in Kenya to report on the 16S rRNA approach in tick microbial diversity.

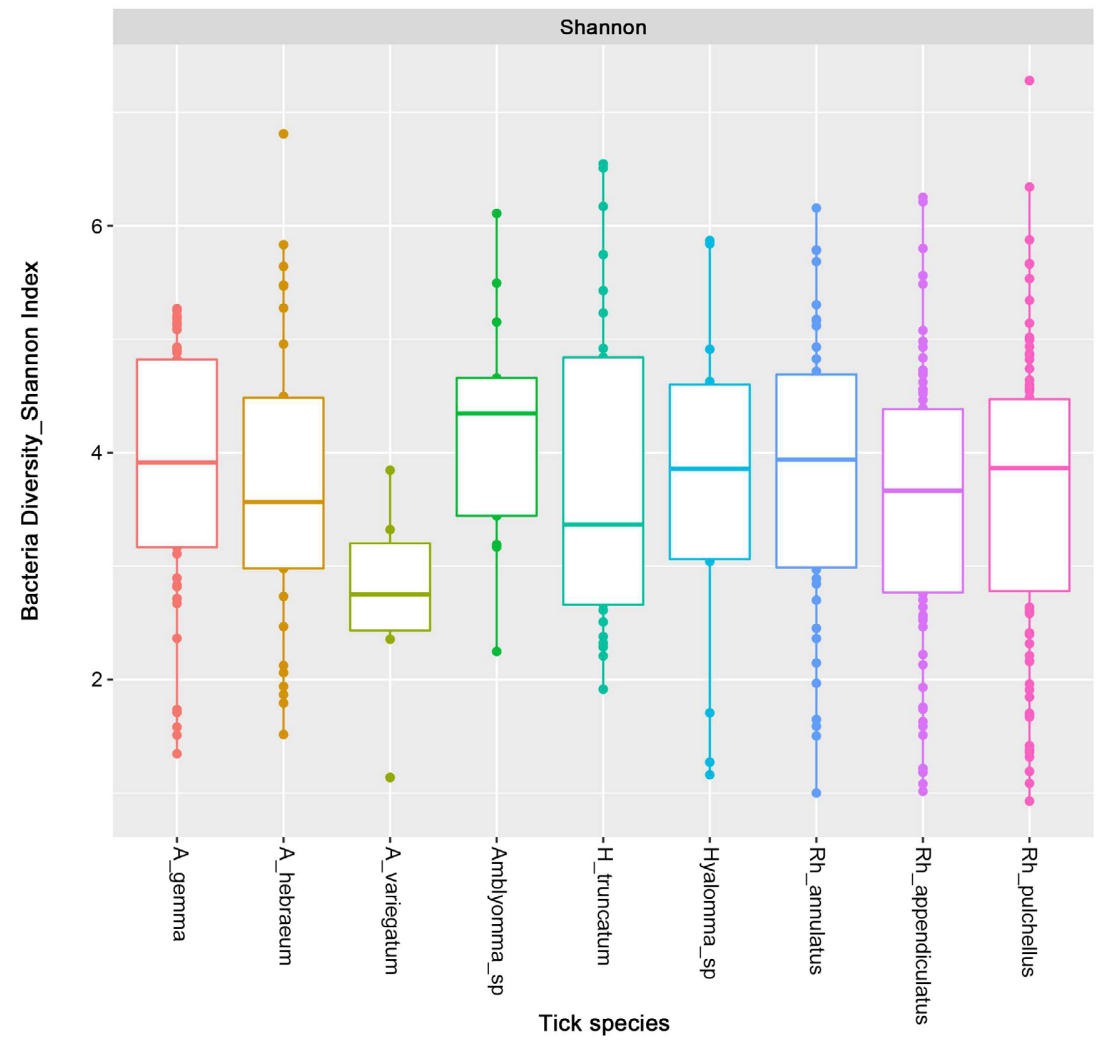

Figure 3. Boxplot showing median bacteria diversity in tick species measured by Shannon diversity index. A. variegatum carried less diverse bacteria (mean Shannon diversity index $(2.69 \pm 0.92$ standard deviation) compared to the other tick species, but the difference was not significant $(\mathrm{p}=0.443)$. 
Four bacteria phyla carried nearly all the tick microbiome (Figure 1). The patterns of bacterial phyla is consistent with findings from other studies that reported over-abundance of Proteobacteria, Firmicutes and Actinobacteria, singly or in different combinations [14] [17] [18] [34] [35]. Of these four, Proteobacteria that comprises Gram negative bacteria, many of them pathogenic to humans and animals [36] [37] accounted for $33.3 \%$ of the OTUs. Firmicutes comprise Gram-positive bacteria that include notable pathogens that are found in different environments [38] and were the second abundant phylum at $23.4 \%$ of the OTUs. Actinobacteria, Gram-positive bacteria that comprise species that are plant and animal pathogens inhabits diverse environments [39] [40] was the third most abundant and contained $20.0 \%$ of the OTUs. Our finding is similar to other reports that indicate Actinobacteria as the third most common phylum after Proteobacteria and Firmicutes [41]. Bacteroidetes was the $4^{\text {th }}$ dominant phylum and constituted $11.6 \%$ of the OTUs. Bacteria in this phylum are Gram negative and inhabit many diverse habitats, where they play a beneficial role in the degradation of organic matter. Nevertheless, some members of the phylum are commonly isolated in human and animal clinical samples, including blood, urine, wounds, and feces [42] [43]. The remaining 23 phyla contributed $11.2 \%$ of the OTUs. Members of these phyla have also been reported in other tick studies [44].

Amongst the 645 genera, three groups were discernible (Figure 2). The biggest group comprised commensals/symbionts (Figure 2, Panel A) that contributed $93.6 \%$ of the genera, but their individual sequence contribution was very low. Group two comprised genera that are known to contain potentially pathogenic species that were probably picked from the livestock during the ticks' blood meals (Figure 2, Panel B). Of these, Coxiella was the most abundant and contributed $41.8 \% \%$ of the total sequence reads. In studies conducted in Malaysia and China, Haemaphysalis ticks were found to contain overabundance of Coxiella [34] [45]. Coxiella also dominated in Amblyomma ticks collected in the United States [46]. Due to inability of the 16S rRNA V3-V4 approach to resolve genera to species, the Coxiella in ticks could contain pathogenic or symbionts species [11]. We opted to error on the side of caution and referred the genera as potentially pathogenic. Corynebacterium in the phylum Actinobacteria, is widely distributed in nature as part of animal and human microbiota, but some species such as $C$. diphtheria cause human infections [40]. This genus was the second most abundant at $13.9 \%$. High abundance of Corynebacterium was found in Hyalomma ticks from United Arab Emirates (UAE) [47]. Other potentially pathogenic bacteria identified included Enterococcus, Staphylococcus, Klebsiella, and Acinetobacter (ESKAPE) that comprise species with high rates of antibiotic resistance [48]. Others included Acinetobacter (4.3\%) and Staphylococcus (3.8\%) and have been reported to occur in Ixodes, Amblyomma and Rhipicephalus ticks [13] [45] [49]. Similarly, Enterococcus and Klebsiella have been reported in low prevalence in ticks collected from dogs in China [13]. Although pathogenic, Burkhoderia, Escherichia-Shegella, Achromobacter, Haemophilus, Legionella, Campylobacter, Treponema, Elizabethkingia, Mycoplasma, Borde- 
tella, Vibrio, and Brucella were present in low abundance. Multiple reports indicate presence of these genera in ticks [13] [44] [50] [51]. Group three comprised genera known to contain TBZ pathogens (Figure 2, Panel B). Rickettsiae, Anaplasma, Francisella, Erlichia, Bartonella and Borellia individually contributed $<1 \%$ of the sequences (Figure 2 Panel C). Unlike other tick microbiome studies that have reported dominance of Rickettsia and Borrelia [14] [18], in this study, these pathogens occurred at $<1 \%$ relative abundance. As shown in Figure 2, panel C, Rickettsia was identified in all tick species accessed but notably in lower prevalence in Hyalomma compared to Amblyomma and Rhipeciphalus. Similar studies in the United Arab Emirates (UAE) and Saudi Arabia have reported absence of Rickettsia in Hyalomma tick collected from camels [47] [52]. Similar to this finding, Anaplasma and Ehrlichia have been reported in relatively low abundance in Tennessee, USA for example [44]. Francisella has also been shown to be dominant in Hyalomma ticks comprising up to $99.1 \%$ in some locations in the UAE [47], but in our study, Francisella was present in much lower abundance (relative abundance of 0.005) and was absent in Hyalomma ticks. Similar to studies in Germany and China [13] [53], Bartonella was detected in low abundance and only in Amblyomma spp and H. truncatum. As a caveat, we cannot say with certainty that the Rickettsia and Francisella identified in this study contained pathogenic species [5] [54] [55]. But, for Rickettsia, we know that Kenyan ticks carry a high prevalence of Rickettsia africae, $R$. aeschlimannii, $R$. mongolotimonae, $R$. conorii subsp. israelensis), Candidatus Rickettsia kulagini, and other unspeciated Rickettsiae [21] [56].

Bacterial community in different tick species varies, probably as a result of environmental factors, blood-meals sources, tick immunity and developmental stages [57]. Contamination at different steps of the DNA extraction, purification and amplification process has been identified as a contributor to the high bacteria diversity seen in ticks [58]. To offset this problem, negative controls are used to track contamination, and if identified, censored from the dataset. Using the "decontam" approached [27], 6 OTUs were dropped from the dataset. Clearly, since the ticks were not surface sterilized before DNA extraction, exo-skeleton associated bacteria contributed to some of the community observed. But, as noted by Narasimhan et al., exo-skeleton associated microbes should be considered as being part of the ticks and they probably play a key role in maintaining a healthy physical immune barrier [59].

As shown in Figure 3, the aggregated mean Shannon diversity index was low (ranged between 2.69 to 4.15), suggesting that the bacteria genera were shared among the tick species. Similar findings have been reported previously and similar to these studies, a few core bacteria taxa, likely endosymbionts dominate [60] [61] [62].

\section{Conclusion}

$16 \mathrm{~S}$ rRNA was used to identify bacterial communities associated with different tick species that were collected from domestic livestock in Kenya. There was lit- 
tle difference in bacteria diversity between the ticks, probably because of similarity in the environment and the hosts that the ticks interact with. Amidst the hundreds of commensal taxa, six genera known to contain TBZ pathogens, namely Rickettsiae, Anaplasma, Francisella, Ehrlichia, Bartonella and Borrelia were identified and illustrate the central role ticks play in "one health" and their usefulness as bio-indicators of pathogens they likely transmit to humans, domestic livestock and wildlife. Future studies should, in addition to prokaryotic $16 \mathrm{~S}$ rRNA, include eukaryotic $18 \mathrm{~S}$ rRNA and viral discovery in order to comprehensively monitor microbial ecosystem in the ticks.

\section{Ethical Statement}

The tick samples were collected under protocol SSC\#1248 that was reviewed and approved by the Animal Use Committee of the Kenya Medical Research Institute.

\section{Acknowledgements}

This work is published with the permission of the Director, Kenya Medical Research Institute. This work was funded by the Armed Forces Health Surveillance Division (AFHSD) and its Global Emerging Infections Surveillance and Research Branch (insert ProMIS ID P0136_19_KY, 2019 and P0095_21_KY, 2021.

\section{Disclaimer}

Material has been reviewed by the Walter Reed Army Institute of Research. There is no objection to its publication. The opinions or assertions contained herein are the private views of the author, and they are not to be construed as official, or as reflecting true views of the Department of the Army or the Department of Defense.

\section{Conflicts of Interest}

The authors declare no conflicts of interest regarding the publication of this paper.

\section{References}

[1] de la Fuente, J., Estrada-Pena, A., Venzal, J.M., Kocan, K.M. and Sonenshine, D.E. (2008) Overview: Ticks as Vectors of Pathogens That Cause Disease in Humans and Animals. Frontiers in Bioscience, 13, 6938-6946. http://www.ncbi.nlm.nih.gov/pubmed/18508706 https://doi.org/10.2741/3200

[2] Raileanu, C., Moutailler, S., Pavel, I., Porea, D., Mihalca, A.D., Savuta, G., et al. (2017) Borrelia Diversity and Co-Infection with Other Tick Borne Pathogens in Ticks. Frontiers in Cellular and Infection Microbiology, 7, 36. http://journal.frontiersin.org/article/10.3389/fcimb.2017.00036/full https://doi.org/10.3389/fcimb.2017.00036

[3] Parola, P. and Raoult, D. (2001) Ticks and Tickborne Bacterial Diseases in Humans: 
An Emerging Infectious Threat. Clinical Infectious Diseases, 32, 897-928.

http://cid.oxfordjournals.org/cgi/content/long/32/6/897

https://doi.org/10.1086/319347

[4] Moutailler, S., Valiente Moro, C., Vaumourin, E., Michelet, L., Tran, F.H., Devillers, E., et al. (2016) Co-Infection of Ticks: The Rule Rather than the Exception. PLOS Neglected Tropical Diseases, 10, e0004539.

https://doi.org/10.1371/journal.pntd.0004539

[5] Bonnet, S.I., Binetruy, F., Hernández-Jarguín, A.M. and Duron, O. (2017) The Tick Microbiome: Why Non-Pathogenic Microorganisms Matter in Tick Biology and Pathogen Transmission. Frontiers in Cellular and Infection Microbiology, 7, 236. https://doi.org/10.3389/fcimb.2017.00236

[6] Motro, Y. and Moran-Gilad, J. (2017) Next-Generation Sequencing Applications in Clinical Bacteriology. Biomolecular Detection and Quantification, 14, 1-6.

https://doi.org/10.1016/j.bdq.2017.10.002

[7] Chakravorty, S., Helb, D., Burday, M., Connell, N. and Alland, D. (2007) A Detailed Analysis of 16S Ribosomal RNA Gene Segments for the Diagnosis of Pathogenic Bacteria. The Journal of Microbiological Methods, 69, 330-339. https://doi.org/10.1016/j.mimet.2007.02.005

[8] Srinivasan, R., Karaoz, U., Volegova, M., MacKichan, J., Kato-Maeda, M., Miller, S., et al. (2015) Use of 16S rRNA Gene for Identification of a Broad Range of Clinically Relevant Bacterial Pathogens. PLoS ONE, 10, e0117617. https://doi.org/10.1371/journal.pone.0117617

[9] Winand, R., Bogaerts, B., Hoffman, S., Lefevre, L., Delvoye, M., Van Braekel, J., et al. (2020) Targeting the 16s rRNA Gene for Bacterial Identification in Complex Mixed Samples: Comparative Evaluation of Second (Illumina) and Third (Oxford Nanopore Technologies) Generation Sequencing Technologies. International Journal of Molecular Sciences, 21, 1-22. https://doi.org/10.3390/ijms21010298

[10] Fuks, G., Elgart, M., Amir, A., Zeisel, A., Turnbaugh, P.J., Soen, Y., et al. (2018) Combining 16S rRNA Gene Variable Regions Enables High-Resolution Microbial Community Profiling. Microbiome, 6, 17. https://doi.org/10.1186/s40168-017-0396-x

[11] Bukin, Y.S., Galachyants, Y.P., Morozov, I.V., Bukin, S.V., Zakharenko, A.S. and Zemskaya, T.I. (2019) The Effect of 16s rRNA Region Choice on Bacterial Community Metabarcoding Results. Scientific Data, 6, Article ID: 190007. https://doi.org/10.1038/sdata.2019.7

[12] Couper, L. and Swei, A. (2018) Tick Microbiome Characterization by Next-Generation 16S rRNA Amplicon Sequencing. Journal of Visualized Experiments, No. 138, 58239. https://doi.org/10.3791/58239

[13] Wang, S., Hua, X. and Cui, L. (2021) Characterization of Microbiota Diversity of Engorged Ticks Collected From Dogs in China. Journal of Veterinary Science, 22, e37. https://doi.org/10.4142/jvs.2021.22.e37

[14] van Treuren, W., Ponnusamy, L., Brinkerhoff, R.J., Gonzalez, A., Parobek, C.M., Juliano, J.J., et al. (2015) Variation in the Microbiota of Ixodes Ticks with Regard to Geography, Species, and Sex. Applied and Environmental Microbiology, 81, 6200-6209. https://doi.org/10.1128/AEM.01562-15

[15] Bouquet, J., Melgar, M., Swei, A., Delwart, E., Lane, R.S. and Chiu, C.Y. (2017) Metagenomic-Based Surveillance of Pacific Coast Tick Dermacentor occidentalis Identifies Two Novel Bunyaviruses and an Emerging Human Ricksettsial Pathogen. Scientific Reports, 7, Article No. 12234. https://doi.org/10.1038/s41598-017-12047-6 
[16] Kisten, D., Brinkerhoff, J., Tshilwane, S.I. and Mukaratirwa, S. (2021) A Pilot Study on the Microbiome of Amblyomma Hebraeum Tick Stages Infected and Non-Infected with Rickettsia africae. Pathogens, 10, 941. https://doi.org/10.3390/pathogens10080941

[17] Hoffmann, A., Müller, T., Fingerle, V. and Noll, M. (2021) Presence of Human Pathogens of the Borrelia Burgdorferi Sensu Lato Complex Shifts the Sequence Read Abundances of Tick Microbiomes in Two German Locations. Microorganisms, 9, 1814. https://doi.org/10.3390/microorganisms9091814

[18] Thapa, S., Zhang, Y. and Allen, M.S. (2019) Bacterial Microbiomes of Ixodes scapularis Ticks Collected from Massachusetts and Texas, USA. BMC Microbiology, 19, 138. https://doi.org/10.1186/s12866-019-1514-7

[19] Smith, T.A., Driscoll, T., Gillespie, J.J. and Raghavan, R. (2015) A Coxiella-Like Endosymbiontis a Potential Vitamin Source for the Lone Star Tick. Genome Biology and Evolution, 7, 831-838. https://doi.org/10.1093/gbe/evv016

[20] Gerhart, J.G., Moses, A.S. and Raghavan, R. (2016) A Francisella-Like Endosymbiont in the Gulf Coast Tick Evolved from a Mammalian Pathogen. Scientific Reports, 6, Article No. 33670. https://doi.org/10.1038/srep33670

[21] Mutai, B.K., Wainaina, J.M., Magiri, C.G., Nganga, J.K., Ithondeka, P.M., Njagi, O.N., et al. (2013) Zoonotic Surveillance for Rickettsiae in Domestic Animals in Kenya. Vector-Borne and Zoonotic Diseases, 13, 360-366. https://doi.org/10.1089/vbz.2012.0977

[22] Estrada-Peña, A. and Jongejan, F. (1999) Ticks Feeding on Humans: A Review of Records on Human-Biting Ixodoidea with Special Reference to Pathogen Transmission Climate, Niche, Ticks, and Models: What They Are and How We Should Interpret Them. Experimental and Applied Acarology, 23, 685-715.

[23] Klindworth, A., Pruesse, E., Schweer, T., Peplies, J., Quast, C., Horn, M., et al. (2013) Evaluation of General 16S Ribosomal RNA Gene PCR Primers for Classical and Next-Generation Sequencing-Based Diversity Studies. Nucleic Acids Research, 41, e1. https://doi.org/10.1093/nar/gks808

[24] Schloss, P.D., Westcott, S.L., Ryabin, T., Hall, J.R., Hartmann, M., Hollister, E.B., et al. (2009) Introducing Mothur: Open-Source, Platform-Independent, Community-Supported Software for Describing and Comparing Microbial Communities. Applied and Environmental Microbiology, 75, 7537-7541.

https://doi.org/10.1128/AEM.01541-09

[25] Quast, C., Pruesse, E., Yilmaz, P., Gerken, J., Schweer, T., Yarza, P., et al. (2013) The SILVA Ribosomal RNA Gene Database Project: Improved Data Processing and Web-Based Tools. Nucleic Acids Research, 41, D590-D596.

https://doi.org/10.1093/nar/gks1219

[26] Weiss, S., Xu, Z.Z., Peddada, S., Amir, A., Bittinger, K., Gonzalez, A., et al. (2017) Normalization and Microbial Differential Abundance Strategies Depend upon Data Characteristics. Microbiome, 5, 27. https://doi.org/10.1186/s40168-017-0237-y

[27] Davis, N.M., Proctor, D.M., Holmes, S.P., Relman, D.A. and Callahan, B.J. (2017) Simple Statistical Identification and Removal of Contaminant Sequences in Marker-Gene and Metagenomics Data. Microbiome, 6, Article No. 226.

https://doi.org/10.1101/221499

[28] McMurdie, P.J. and Holmes, S. (2013) Phyloseq: An R Package for Reproducible Interactive Analysis and Graphics of Microbiome Census Data. PLoS ONE, 8, e61217. https://doi.org/10.1371/journal.pone.0061217

[29] Oksanen, J., Blanchet, F.G., Kindt, R., Legendre, P., Minchin, P.R., O’Hara, R.B., 
Simpson, G.L., Solymos, P., Stevens, M.H.H. and Wagner, H. (2014) Vegan: Community Ecology Package. R Package Version 2.2-0.

[30] Wickham, H. (2009) ggplot2: Elegant Graphics for Data Analysis. Springer-Verlag, New York. https://doi.org/10.1007/978-0-387-98141-3

[31] R Core Team (2018) R: A Language and Environment for Statistical Computing. R Foundation for Statistical Computing, Vienna. http://www.gbif.org/resource/81287

[32] Weiss, S., Xu, Z., Amir, A., Peddada, S., Bittinger, K., Gonzalez, A., et al. (2015) Effects of Library Size Variance, Sparsity, and Compositionality on the Analysis of Microbiome Data. PeerJ Preprints, 3, e1408. https://doi.org/10.7287/peerj.preprints.1157v1

[33] Shannon, C.E. (1948) A Mathematical Theory of Communication. The Bell System Technical Journal, 27, 623-656. https://doi.org/10.1002/j.1538-7305.1948.tb00917.x

[34] Zhang, R.L., Huang, Z.D., Yu, G.F. and Zhang, Z. (2019) Characterization of the Bacterial Community in Haemaphysalis longicornis (Acari: Ixodidae) throughout Developmental Stages. Experimental and Applied Acarology, 77, 173-186. https://doi.org/10.1007/s10493-019-00339-7

[35] Budachetri, K., Browning, R.E., Adamson, S.W., Dowd, S.E., Chao, C.-C., Ching, W.-M., et al. (2014) An Insight into the Microbiome of the Amblyomma maculatum (Acari: Ixodidae) NIH Public Access. Journal of Medical Entomology, 51, 119-129. https://doi.org/10.1603/ME12223

[36] Gupta, R.S. (2000) The Phylogeny of Proteobacteria: Relationships to Other Eubacterial Phyla and Eukaryotes. FEMS Microbiology Reviews, 24, 367-402. https://doi.org/10.1111/j.1574-6976.2000.tb00547.x

[37] Stackebrandt, E., Murray, R.G.E. and Truper, H.G. (1988) Proteobacteria classis nov., a Name for the Phylogenetic Taxon That Includes the "Purple Bacteria and Their Relatives". International Journal of Systematic Bacteriology, 38, 321-325. https://doi.org/10.1099/00207713-38-3-321

[38] Galperin, M.Y. (2015) Genome Diversity of Spore-Forming Firmicutes Bacterial Systematics from Gram Stain to $16 \mathrm{~S}$ rRNA. Microbiology Spectrum, 1, 1-27. https://www.ncbi.nlm.nih.gov/pmc/articles/PMC4306282/pdf/nihms653814.pdf

[39] Shivlata, L. and Satyanarayana, T. (2015) Thermophilic and Alkaliphilic Actinobacteria: Biology and Potential Applications. Frontiers in Microbiology, 6, Article No. 1014. https://doi.org/10.3389/fmicb.2015.01014

[40] Barka, E.A., Vatsa, P., Sanchez, L., Nathalie Gaveau-Vaillant, C.J., Klenk, H.-P., Clément, C., et al. (2016) Taxonomy, Physiology, and Natural Products of Actinobacteria. Microbiology and Molecular Biology Reviews, 80, 1-43. https://doi.org/10.1128/MMBR.00019-15

[41] He, X.M. and Cheng, T.Y. (2018) Tick Saliva Microbiomes Isolated from Engorged and Partially Fed Adults of Haemaphysalis flava Tick Females. Journal of Applied Entomology, 142, 173-180. https://doi.org/10.1111/jen.12427

[42] Thomas, F., Hehemann, J.H., Rebuffet, E., Czjzek, M. and Michel, G. (2011) Environmental and Gut Bacteroidetes: The Food Connection. Frontiers in Microbiology, 2, 93. https://doi.org/10.3389/fmicb.2011.00093

[43] Hugo, C.J., Jooste, P.J., Segers, P., Vancanneyt, M. and Kersters, K. (1999) A Polyphasic Taxonomic Study of Chryseobacterium Strains Isolated from Dairy Sources. Systematic and Applied Microbiology, 22, 586-595.

https://doi.org/10.1016/S0723-2020(99)80012-5

[44] Trout Fryxell, R.T. and DeBruyn, J.M. (2016) The Microbiome of Ehrlichia-Infected 
and Uninfected Lone Star Ticks (Amblyomma americanum). PLoS ONE, 11, e0155559. https://doi.org/10.1371/journal.pone.0146651

[45] Khoo, J.J., Lim, F.S., Chen, F., Phoon, W.H., Khor, C.S., Pike, B.L., et al. (2016) Coxiella Detection in Ticks from Wildlife and Livestock in Malaysia. Vector-Borne and Zoonotic Diseases, 16, 744-751. https://doi.org/10.1089/vbz.2016.1959

[46] Clay, K. and Fuqua, C. (2010) The Tick Microbiome: Diversity, Distribution and Influence of the Internal Microbial Community for a Blood-Feeding Disease Vector. Critical Needs and Gaps in Understand Prevention, Amelioration, and Resolution of Lyme and Other Tick-Borne Diseases: the Short-Term and Long-Term Outcomes. Institute of Medicine Committee on Lyme Disease and Other Tick-Borne Diseases: The State of the Science, Washington DC, 1-22.

[47] Perveen, N., Muzaffar, S.B., Vijayan, R. and Al-Deeb, M.A. (2020) Microbial Communities Associated with the Camel Tick, Hyalomma dromedarii: 16S rRNA Gene-Based Analysis. Scientific Reports, 10, Article No. 17035. https://doi.org/10.1038/s41598-020-74116-7

[48] Santajit, S. and Indrawattana, N. (2016) Mechanisms of Antimicrobial Resistance in ESKAPE Pathogens. BioMed Research International, 2016, Article ID: 2475067. https://doi.org/10.1155/2016/2475067

[49] Jory Brinkerhoff, R., Clark, C., Ocasio, K., Gauthier, D.T. and Hynes, W.L. (2020) Factors Affecting the Microbiome of Lxodes scapularis and Amblyomma americanum. PLoS ONE, 15, e0232398. https://doi.org/10.1371/journal.pone.0232398

[50] Carpi, G., Cagnacci, F., Wittekindt, N.E., Zhao, F., Qi, J., Tomsho, L.P., et al. (2011) Metagenomic Profile of the Bacterial Communities Associated with Ixodes ricinus Ticks. PLoS ONE, 6, e25604. https://doi.org/10.1371/journal.pone.0025604

[51] Andreotti, R., De León, A.A.P., Dowd, S.E., Guerrero, F.D., Bendele, K.G. and Scoles, G.A. (2011) Assessment of Bacterial Diversity in the Cattle Tick Rhipicephalus (Boophilus) Microplus through Tag-Encoded Pyrosequencing. BMC Microbiology, 11, Article No. 6. http://www.biomedcentral.com/1471-2180/11/6 https://doi.org/10.1186/1471-2180-11-6

[52] Elbir, H., Almathen, F. and Alhumam, N.A. (2019) A Glimpse of the Bacteriome of Hyalomma dromedarii Ticks Infesting Camels Reveals Human Helicobacter pylori Pathogen. The Journal of Infection in Developing Countries, 13, 1001-1012. https://doi.org/10.3855/jidc.11604

[53] Regier, Y., Komma, K., Weigel, M., Kraiczy, P., Laisi, A., Pulliainen, A.T., et al. (2019) Combination of Microbiome Analysis and Serodiagnostics to Assess the Risk of Pathogen Transmission by Ticks to Humans and Animals in Central Germany 11 Medical and Health Sciences 1108 Medical Microbiology. Parasites and Vectors, 12, Article No. 11. https://doi.org/10.1186/s13071-018-3240-7

[54] Zhang, Y.K., Yu, Z.J., Wang, D., Bronislava, V., Branislav, P. and Liu, J.Z. (2019) The Bacterial Microbiome of Field-Collected Dermacentor marginatus and Dermacentor reticulatus from Slovakia. Parasites and Vectors, 12, Article No. 325. https://doi.org/10.1186/s13071-019-3582-9

[55] Špitalská, E., Sparagano, O., Stanko, M., Schwarzová, K., Špitalský, Z., Škultéty, L., et al. (2018) Diversity of Coxiella-Like and Francisella-Like Endosymbionts, and Rickettsia spp., Coxiella burnetii as Pathogens in the Tick Populations of Slovakia, Central Europe. Ticks and Tick-Borne Diseases, 9, 1207-1211. https://doi.org/10.1016/j.ttbdis.2018.05.002

[56] Kimita, G., Mutai, B., Nyanjom, S.G., Wamunyokoli, F. and Waitumbi, J. (2016) Phylogenetic Variants of Rickettsia africae, and Incidental Identification of "Candi- 
datus Rickettsia Moyalensis” in Kenya. PLOS Neglected Tropical Diseases, 10, e0004788. https://doi.org/10.1371/journal.pntd.0004788

[57] Wu-Chuang, A., Hodžić, A., Mateos-Hernández, L., Estrada-Peña, A., Obregon, D. and Cabezas-Cruz, A. (2021) Current Debates and Advances in Tick Microbiome Research. Current Research in Parasitology \& Vector-Borne Diseases, 1, Article ID: 100036. https://doi.org/10.1016/j.crpvbd.2021.100036

[58] Lejal, E., Estrada-Peña, A., Marsot, M., Cosson, J.F., Rué, O., Mariadassou, M., et al. (2020) Taxon Appearance from Extraction and Amplification Steps Demonstrates the Value of Multiple Controls in Tick Microbiota Analysis. Frontiers in Microbiology, 11, Article No. 1093. https://doi.org/10.3389/fmicb.2020.01093

[59] Narasimhan, S. and Fikrig, E. (2015) Tick Microbiome: The Force Within. Trends in Parasitology, 31, 315-323. https://doi.org/10.1016/j.pt.2015.03.010

[60] Ross, B.D., Hayes, B., Radey, M.C., Lee, X., Josek, T., Bjork, J., et al. (2018) Ixodes scapularis Does Not Harbor a Stable Midgut Microbiome. The ISME Journal, 12, 2596-2607. https://doi.org/10.1038/s41396-018-0161-6

[61] Couper, L.I., Kwan, J.Y., Ma, J. and Swei, A. (2019) Drivers and Patterns of Microbial Community Assembly in a Lyme Disease Vector. Ecology and Evolution, 9, 7768-7779. https://doi.org/10.1002/ece3.5361

[62] Guizzo, M.G., Parizi, L.F., Nunes, R.D., Schama, R., Albano, R.M., Tirloni, L., et al. (2017) A Coxiella Mutualist Symbiont Is Essential to the Development of Rhipicephalus microplus. Scientific Reports, 7, Article No. 17554.

https://doi.org/10.1038/s41598-017-17309-x 